\title{
Absence of parasympathetic denervation of the iris in alcoholics
}

\author{
W I L L I A M M Y R S, K E N NETH W I L LIS, \\ A ND A LEX A NDER REEVES \\ From the David Prosser Neurology Research Laboratories, Section of Neurology, \\ Dartmouth-Hitchcock Medical Center, Hanover, New Hampshire, USA
}

S UMMARY Forty patients with a history of alcohol abuse were treated with a $2 \%$ solution of methacholine to determine the prevalence of iridic parasympathetic denervation. Three $(8 \%)$ of the alcoholics showed iris constriction of $1.0 \mathrm{~mm}$ or more, compared with three $(8 \%)$ control subjects. In contrast, damage to the iridic parasympathetic nerve supply occurs in approximately $80 \%$ of patients with diabetes mellitus as determined by the same pharmacological technique.

Peripheral neuropathy is a well-known complication of chronic excessive ethanol consumption associated with dietary deficiency. However, symptomatic involvement of the autonomic nervous system is unusual in alcoholics. Novak and Victor (1974) have reported three severely ill patients with dysphonia and dysphagia who at necropsy showed degeneration of the vagus nerves. Excessive or absent sweating of the feet may also be seen in alcoholics with polyneuropathy.

In diabetes mellitus, somatic neuropathy may occur early and may even be present before other manifestations of disordered carbohydrate metabolism. Involvement of the autonomic nervous system has also been shown to occur early in the course of the disease (Martin, 1953). Parasympathetic denervation hypersensitivity of the iris to $2 \%$ methacholine was found in $74 \%$ of diabetics diagnosed within the preceding two years (Sigsbee et al., 1974). The sensitivity of the methacholine test in showing early parasympathetic denervation of the iris in diabetes mellitus led us to study alcoholics to determine the prevalence of iridic denervation in that population.

\section{Subjects and methods}

Forty male alcoholic patients in the White River Junction VA Alcohol Rehabilitation Program were

Address for reprint requests: Dr A. G. Reeves, Section of Neurology, Department of Medicine, Dartmouth Medical School, Hanover, New Hampshire 03755, USA.

Accepted 23 April 1979 studied. Heavy metal exposure, cancer, uraemia, thyroid disease, and the use of neurotoxic drugs were reasons for exclusion from the study. All patients had normal fasting blood glucose levels at the time of study.

Peripheral nerve examination consisted of testing sensory modalities, motor function, and deep tendon reflexes. The autonomic nervous system was evaluated for orthostatic changes in blood pressure and heart rate, for sweating abnormality by the Valsalva manoeuvre, and by the metacholine test, as described by Sigsbee et al. (1974). Thirty-six subjects from the staff and patients of the Mary Hitchcock Memorial Hospital and Strong Memorial Hospital served as control subjects and have been reported previously (Sigsbee et al., 1974).

\section{Results}

Three patients $(8 \%)$ showed a positive response to methacholine. In all three the pupillary constriction was $1 \mathrm{~mm}$ or greater in each eye. All three responders had absent ankle reflexes and decreased vibration perception in the feet. Twenty-four $(60 \%)$ patients had no evidence of peripheral neuropathy. Sixteen $(40 \%)$ had depressed ankle reflexes-10 of these 16 showed decreased vibration perception in the feet, and two also had evidence of distal weakness in the lower extremities. Decreased sweating of the feet was found in six patients. No orthostatic hypotension, blunting of the Valsalva response, dysphonia, or dysphagia were observed. 


\section{Discussion}

We have shown that alcoholics do not develop iridic denervation more frequently than a control population. In contrast, the methacholine test has shown iridic denervation hypersensitivity in 50 of $62(81 \%)$ diabetics, many of whom were recently diagnosed and asymptomatic (Sigsbee et al., 1974). Within one year of a positive methacholine test, one of our alcoholic patients developed chemical diabetes. Perhaps some of the other people with miotic responses to methacholine, alcoholics and control subjects, may have had chemical or undiagnosed diabetes mellitus. In the two studies in which control populations were studied with methacholine, the control subjects were not screened with blood glucose measurements or glucose tolerance tests (Brunnschweiler, 1954; Sigsbee et al., 1974). We are currently studying glucose tolerance in a general population with positive methacholine responses.

Symptomatic autonomic dysfunction is not common in alcoholics, unless they have severe underlying somatic neuropathy. Low et al. (1975) found anhidrosis of the feet in all of eight patients and an abnormal Valsalva response in two of nine patients with a history of chronic alcohol abuse, but no other autonomic dysfunction was detected. Winship et al. (1968) reported that motility may be impaired in the distal two-thirds of the oesophagus in patients with alcoholic peripheral neuropathy, compared with that motility in similar alcoholics without neuropathy. They speculated that vagal dysfunction, perhaps combined with a myopathic component, might account for the disordered motility pattern. Novak and Victor (1974) reported four alcoholic patients with severe endstage alcoholic disease in whom hoarseness and dysphagia were prominent symptoms. Three of these patients died during their hospitalisation, and postmortem examination showed severe degeneration of the vagus nerves comparable to the changes seen in their peripheral nerves. Although central nervous system lesions characteristic of Wernicke's disease were seen, these lesions were thought to be insufficient to account for the degree of degeneration in the vagus nerves.

We cannot determine from our study why diabetics and alcoholics should demonstrate such different autonomic syndromes. Presumably diabetes mellitus and most of the other chronic disorders with prominent autonomic symptoms have relatively unremitting metabolic or hereditary conditions which impair the normal function of the neurone or Schwann cell. On the other hand, alcoholic neuropathy is thought to be the result of a deficiency of thiamine and other B vitamins. Alcoholics have several reasons to be deficient in thiamine-poor intake, poor absorption, and poor utilisation-when excess ethanol is being processed by the cell. Since improved diet can improve clinical manifestations of alcoholic neuropathy (Strauss, 1935; Walsh and McLeod, 1970), the alcoholic who is occasionally adequately fed should be able to overcome a temporary vitamin deficiency. Thus, denervation would only occur in the most prolonged cases of malnutrition.

William Myers was supported by a grant from the Hitchcock Foundation.

\section{References}

Brunnschweiler, H. (1954). Zur Differentialdiagnose der Pupillotonie unter besonderer Berücksichtigung des Mecholyltests. Confinia Neurologica, 14, 50-64.

Low, P. A., Walsh, J. C., Huang, C. Y., and McLeod, J. G. (1975). The sympathetic nervous system in alcoholic neuropathy: a clinical and pathologic study. Brain, 98, 357-364.

Martin, M. M. (1953). Diabetic neuropathy: a clinical study of 150 cases. Brain, 76, 594-624.

Novak, D. J., and Victor, M. (1974). The vagus and sympathetic nerves in alcoholic polyneuropathy. Archives of Neurology (Chicago), 30, 273-284.

Sigsbee, B., Torkelson, R., Kadis, G., Wright, J. W., and Reeves, A. G. (1974). Parasympathetic denervation of the iris in diabetes mellitus. Journal of Neurology, Neurosurgery, and Psychiatry, 37, 10311035.

Strauss, M. B. (1935). Etiology of alcoholic polyneuritis. American Journal of the Medical Sciences, 189, 378-382.

Walsh, J. C., and McLeod, J. G. (1970). Alcoholic neuropathy: an electrophysiological and histological study. Journal of the Neurological Sciences, $10,457-469$.

Winship, D. H., Caflisch, C. R., Zboralske, F. F., and Hogan, W. J. (1968). Deterioration of esophageal peristalsis in patients with alcoholic neuropathy. Gastroenterology, 55, 173-178. 